OPEN ACCESS

Edited by:

Vin-Cent Wu,

National Taiwan University, Taiwan

Reviewed by:

Shao-Yu Yang,

National Taiwan University Hospital,

Taiwan

Leay Kiaw Er,

Taipei Tzu Chi Hospital, Taiwan

*Correspondence:

Tetsuo Nishikawa

tetsuon@yokohamah.johas.go.jp

Specialty section:

This article was submitted to

Neuroendocrine Science,

a section of the journal

Frontiers in Endocrinology

Received: 23 December 2020

Accepted: 15 February 2021

Published: 16 March 2021

Citation:

Inoue $K$, Kitamoto $T$, Tsurutani $Y$,

Saito J, Omura M and Nishikawa T

(2021) Cortisol Co-Secretion and

Clinical Usefulness of ACTH

Stimulation Test in Primary

Aldosteronism: A Systematic Review and Biases in Epidemiological Studies.

Front. Endocrinol. 12:645488.

doi: 10.3389/fendo.2021.645488

\section{Cortisol Co-Secretion and Clinical Usefulness of ACTH Stimulation Test in Primary Aldosteronism: A Systematic Review and Biases in Epidemiological Studies}

\author{
Kosuke Inoue ${ }^{1,2}$, Takumi Kitamoto ${ }^{2,3}$, Yuya Tsurutani ${ }^{2}$, Jun Saito ${ }^{2}$, Masao Omura $^{2}$ \\ and Tetsuo Nishikawa ${ }^{2 *}$

\begin{abstract}
${ }^{1}$ Department of Epidemiology, University of California, Los Angeles (UCLA) Fielding School of Public Health, Los Angeles, CA, United States, ${ }^{2}$ Endocrinology and Diabetes Center, Yokohama Rosai Hospital, Yokohama, Japan, ${ }^{3}$ Division of Endocrinology, Department of Medicine, Columbia University, New York, NY, United States
\end{abstract}

The hypothalamus-pituitary-adrenal (HPA) axis plays an important role in primary aldosteronism. Aldosterone biosynthesis is regulated not only by angiotensin II in the renin-angiotensin-aldosterone system, but also by adrenocorticotropic hormone (ACTH), one of the key components of the HPA axis. Although previous studies have reported cortisol cosecretion in primary aldosteronism, particularly aldosterone-producing adenoma (APA), the clinical relevance of such aldosterone and cortisol cosecretion from APA and hypertension or other metabolic disorders has not been fully established. Several somatic mutations including KCNJ5 and CACNA1D are known to induce autonomous production of aldosterone in APA, and the aldosterone responsiveness to ACTH may vary according to each mutation. The ACTH stimulation test has been reported to be a useful tool to distinguish the subtypes of primary aldosteronism (e.g., unilateral vs bilateral) in some studies, but it has not been commonly applied in clinical practice due to limited evidence. Given the recent advancement of imaging, omics research, and computational approach, it is important to summarize the most updated evidence to disentangle the potential impact of cortisol excess in primary aldosteronism and whether the ACTH stimulation test needs to be considered during the diagnostic process of primary aldosteronism. In this article, we conducted a systematic review of epidemiological studies about (i) cortisol cosecretion in primary aldosteronism and (ii) the ACTH stimulation test for the diagnosis of primary aldosteronism (including subtype diagnosis). Then, we discussed potential biases (e.g., confounding bias, overadjustment, information bias, selection bias, and sampling bias) in the previous studies and introduced some advanced epidemiological/statistical methods to minimize these limitations. A better understanding of biases and epidemiological perspective on this topic would allow us to produce further robust evidence and balanced discussion about the causal mechanisms involving the HPA axis and clinical usefulness of the ACTH stimulation test among patients with primary aldosteronism.

Keywords: cortisol, ACTH, primary aldosteronism, systematic review, bias, epidemiological methods 


\section{INTRODUCTION}

Primary aldosteronism (PA) is one of the common causes of secondary hypertension, increasing the risk of cardiovascular disease (CVD) and renal events (1-4). Since the first report of a patient with adrenal adenoma concurrently producing aldosterone and cortisol (5), cortisol cosecretion in PA has received substantial attention, particularly because it may cause severe complications due to cortisol excess in addition to aldosterone excess. A recent study using a mass spectrometrybased analysis in Europe showed that cortisol cosecretion in PA was associated with adverse metabolic risk factors (6), indicating the role of glucocorticoid on an increased risk of metabolic disorders among patients with PA (7-11). Moreover, while the actual prevalence of this phenotype is not clear, some studies have suggested a relatively high prevalence of PA with hypercortisolism than expected $(6,10-15)$. Therefore, it is imperative to understand the current state of knowledge about cortisol cosecretion in PA, and consider whether the evaluation of cortisol excess needs be recommended in general PA screening or not.

Although the hypothalamus-pituitary-adrenal (HPA) axis plays an important role in regulating aldosterone biosynthesis, the clinical usefulness of ACTH stimulation test during the management of PA, particularly for PA subtype diagnosis (e.g., aldosterone-producing adenoma [APA] vs. bilateral aldosterone hyperplasia $[\mathrm{BAH}])$, has been unclear. In general, ACTH is known to stimulate both cortisol and aldosterone secretion acutely and transiently through binding to melanocortin type 2 receptor (MC2R) (16). Previous studies have shown the high performance of classifying subtype of PA based on plasma aldosterone concentrations during ACTH stimulation test under 1-mg dexamethasone suppression test (DST) $(17,18)$. Other studies have also reported a larger decrease in aldosterone levels following dexamethasone suppression of HPA axis among APA than BAH (19) or among KCNJ5-mutated APAs than KCNJ5 wild-type APAs (20), indicating the regulating role of endogenous ACTH on aldosterone secretion among patients with PA. Because lateralization of hyperaldosteronism is crucial to determine the target treatment (i.e., adrenalectomy or medication therapy) (21), there has been a great interest in the clinical benefit of conducting ACTH stimulation test during the PA confirmation process before AVS - a gold standard but more invasive and challenging procedure for PA subtype diagnosis.

Over the last several decades, observational studies have played a key role in PA research. Because exposure or treatment is not randomized in observational studies, we always need to be careful about confounding bias, a bias due to unmeasured common causes of exposure and outcomes (22). This is also the case in the "big data" analysis or machine learning-based approach because they do not guarantee the adjustment of a sufficient set of confounders and could rather introduce precisely wrong conclusion due to small variance of the estimates under the presence of bias. Moreover, both observational studies and randomized controlled trials suffer from other sources of bias including information bias (e.g., measurement error of hormone levels, self-report of history of hypertension and medications) (23) and selection bias (e.g., loss to follow-up, selection of study sample based on both exposure and outcome assessment) (24). Limited generalizability due to sampling bias is another issue of previous PA-related studies because most of the studies have been conducted at single-center and/or specific cohorts.

This review has two goals. First, we will provide updated summaries of epidemiological studies about cortisol cosecretion in primary aldosteronism and the clinical usefulness of the ACTH stimulation test to diagnose primary aldosteronism and/or its subtypes. Then, we will explain possible biases (e.g., confounding bias, overadjustment, information bias, selection bias, and sampling bias) in these epidemiological studies and some methods to minimize such limitations in future PA-related studies. Recognizing these biases and applying the advanced epidemiological methods would help us to build a further balanced and profound discussion on this topic-cortisol cosecretion and clinical usefulness of the ACTH stimulation test in PA.

\section{CORTISOL COSECRETION IN PRIMARY ALDOSTERONISM}

Recently, there is increasing literature regarding cortisol cosecretion in PA and its impact on CVD risk factors $(6,25,26)$. We conducted literature searches between January 2000 and November 2020 using the electronic databases MEDLINE and EMBASE for cohort studies investigating the cortisol cosecretion in primary aldosteronism. The following search terms were applied: ("cortisol" OR "cortisolemia" OR "Cushing”) AND ("primary aldosteronism” OR "aldosteroneproducing adenoma”). We extracted the following information: first author name, publication year, region of study, populations, exposures/comparators, outcomes, and study design. We restricted studies to those with a sample size $\geq 10$ (to avoid case reports) and written in English. Flow of studies through review and summary of the identified 16 studies are shown in Figure 1A and Table $1(6,10-15,27-35)$.

Previous studies have shown a relatively high prevalence of subclinical Cushing's syndrome or subclinical hypercortisolemia among patients with PA although the definition of hypercortisolemia differs across studies and countries (Table 1). In 2009, Piaditis et al. reported $12.1 \%$ of patients with adrenal incidentalomas had PA with hypercortisolemia (36). Since then, several studies from the Asian cohort have reported a 5\%-27\% prevalence of subclinical hypercortisolemia among patients with $\mathrm{PA}$ or APA (11-15). A recent multicenter cohort study in Germany showed even $77.6 \%$ of subclinical hypercortisolemia among 161 patients with PA (10). While the difference in clinical characteristics between PA patients with and without hypercortisolemia is still not clear (mainly due to the small sample size in each previous study) and may vary by geographical area and definitions, most of these studies showed larger tumor size among PA patients with subclinical hypercortisolemia than those without.

In 2014, monoclonal antibodies against human CYP11B1 and CYP11B2 were developed and their expression was reported in APAs, indicating the cortisol synthesis in such adrenal tumors $(37,38)$. This finding was supported by a small cohort study 
A

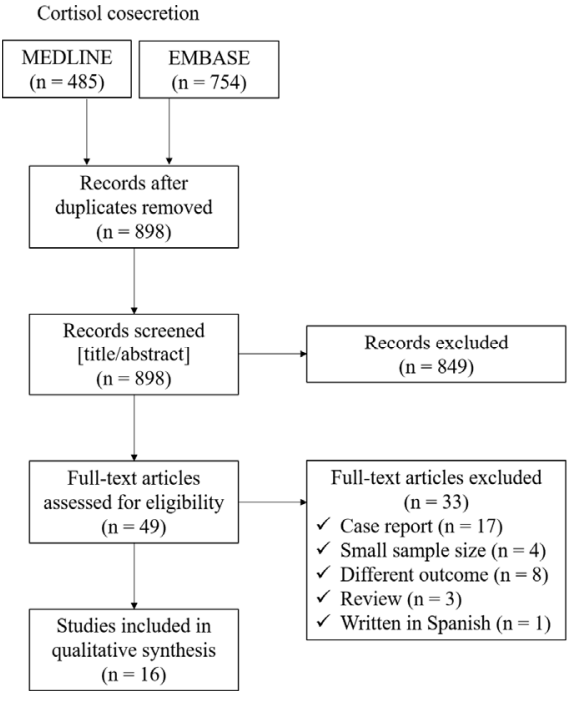

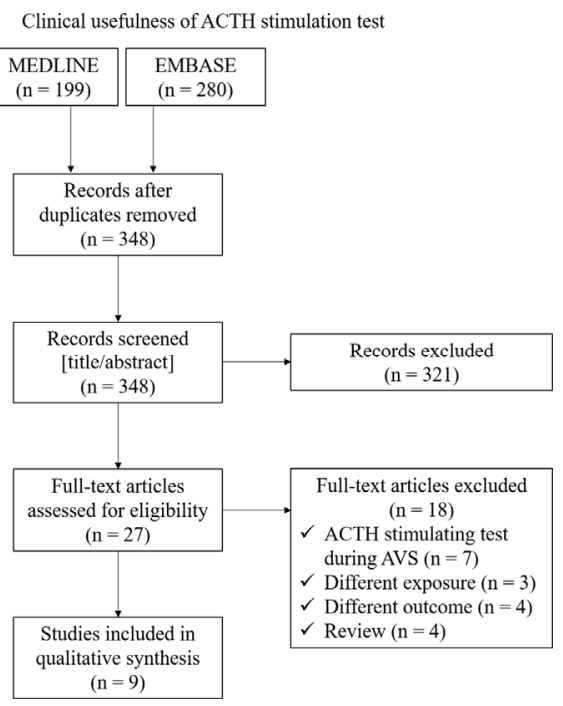

FIGURE 1 | Flow of studies through review for (A) cortisol cosecretion and (B) clinical usefulness of ACTH stimulation test in primary aldosteronism.

reporting a significant decrease in serum cortisol levels during 1 mg DST after the resection of APAs (29) and a large mass spectrometry-based analysis showing higher excretion of cortisol and total glucocorticoid metabolites in the PA group compared with the healthy control group (6). Moreover, a previous case series showed that the majority of APAs with subclinical hypercortisolemia was composed mainly of ZF-like cells (39). Given that ZF comprise larger cells that synthesize glucocorticoids compared with ZG that synthesize aldosterone (40), such histopathological feature may be related to the large tumor size in APAs with subclinical hypercortisolemia, that requires further investigation.

Recent studies also have reported health outcomes related to autonomous cortisol secretion in APAs. The above-mentioned mass spectrometry-based analysis also found the correlation between glucocorticoid output and several metabolic markers including body mass index, waist circumference, insulin resistance, high-density lipoprotein, and diastolic blood pressure (6). Tang et al. showed higher proportions of cardiovascular complications, glucose intolerance/diabetes, and osteopenia/ osteoporosis among patients with APAs and cortisol producing adenoma (CPA) than patients with pure APAs (14). Adolf et al. scrutinized the possible impact of cortisol excess on cardiac remodeling among PA patients (30). Across patients in the German Conn's registry, they found the association of total glucocorticoid excretion with the decrease in LVMI a year after the initiation of PA treatment (30). The association between subclinical hypercortisolemia and impaired glucose metabolism in patients with PA was also reported by other cohort studies (10, 11). These results support the adverse effect of glucocorticoid excess on health through the activation of not only glucocorticoid receptor but also mineralocorticoid receptor (MR) through the impaired conversion of cortisol to its MR-inactive cortisone (41). Another study using the same German registry showed significant increases in anti-TPO levels after adrenalectomy in unilateral PA patients with cortisol cosecretion but the trend was not found in unilateral PA patients without cortisol cosecretion and bilateral PA patients receiving mineralocorticoid antagonist therapy (34). Their findings generate the hypothesis that cortisol cosecretion among unilateral PA may exhibit the immunosuppressive effect. Given these findings, it would be important to evaluate the cortisol cosecretion among PA patients in routine clinical practice. To produce robust evidence, more research is warranted to clarify whether the adverse health outcomes of APAs with subclinical hypercortisolemia differ between those with and without CPA.

The possible influence of mild cortisol excess in PA on the interpretation of AVS has been suggested in prior literature. In general, cannulation success is evaluated by selectivity index (the ratio of cortisol concentration for each adrenal vein and inferior vena cava [IVC]), and the lateralization is evaluated by lateralization index (the aldosterone to cortisol ratio on the dominant side with excess aldosterone secretion over aldosterone to cortisol ratio on the non-dominant side) and contralateral suppression index (the aldosterone to cortisol ratio on the non-dominant side over aldosterone to cortisol ratio in IVC) (21). Based on the findings from AVS in patients with CPA, but without PA, Goupil et al, suggested that autonomous unilateral cortisol hypersecretion could confound the accuracy of AVS by affecting the aldosterone/cortisol ratio in the adrenal vein (i.e., selectivity index, lateralization index, and contralateral suppression index), leading to misdiagnosis and suboptimal management (42). However, a recent single-center study focusing on PA with mildly elevated cortisol levels (mean serum cortisol levels during the $1-\mathrm{mg}$ DST $=3.3 \mu \mathrm{g} / \mathrm{dl}$ ) showed that the rates of cannulation success or lateralization did not differ between PA patients with mild cortisol excess and those without cortisol excess during AVS with ACTH stimulation (blood sample was obtained $1 \mathrm{~h}$ after the stimulation) (35). A multi-center cohort study on this topic would help us to validate their findings and 
TABLE 1 | Summary of epidemiological studies about cortisol cosecretion in primary aldosteronism (PA).

\begin{tabular}{|c|c|c|c|c|c|c|}
\hline Author & Year & Region & Populations & Exposures/Comparators & Characteristics/Outcomes & Study design \\
\hline Hiraishi et al. (12) & 2011 & Japan & 38 patients with PA & Coexistence of PA and subclinical Cushing's syndrome ${ }^{a}(n=8)$ & Clinical and histopathological characteristics & Cross-sectional study \\
\hline Nakajima et al. (27) & 2011 & Japan & 76 patients with PA & $\begin{array}{l}\text { Coexistence of PA and subclinical cortisol hypersecretion ( } \mathrm{n}=22 \text {, } \\
\text { serum cortisol levels during } 1 \mathrm{mg} \text { DST } \geq 3.0 \mu \mathrm{g} / \mathrm{dl})\end{array}$ & $\begin{array}{l}\text { Clinical characteristics including a history of } \\
\text { cardiovascular events }\end{array}$ & Cross-sectional study \\
\hline Fallo et al. (28) & 2011 & Italy & 76 patients with PA & $\begin{array}{l}\text { Coexistence of PA and subclinical cortisol hypersecretion ( } \mathrm{n}=3 \text {, serum } \\
\text { cortisol levels during } 1 \mathrm{mg} \mathrm{DST}>1.8 \mu \mathrm{g} / \mathrm{dl} \text { ) }\end{array}$ & Clinical and histopathological characteristics & Cohort study \\
\hline Fujimoto et al. (13) & 2013 & Japan & 39 PA patients & Coexistence of PA and subclinical Cushing's syndrome $e^{a}(n=5)$ & Clinical and histopathological characteristics & Cross-sectional study \\
\hline Arlt et al. (6) & 2017 & Germany & 174 patients with $P A^{b, c}$ & $\begin{array}{l}\text { Cortisol cosecretion (24-h cortisol and total glucocorticoid outputs } \\
\text { collected by quantitative gas chromatography-mass spectrometry) }\end{array}$ & $\begin{array}{l}\text { Metabolic risk factors (BMI, blood pressure, fasting } \\
\text { plasma glucose and insulin, 2-h glucose values during } \\
\text { 75-g oral glucose tolerance test, HbA1c, total } \\
\text { cholesterol, HDL, and triglycerides) }\end{array}$ & Cohort study \\
\hline Inoue et al. (29) & 2017 & Japan & $\begin{array}{l}30 \text { patients with APA } \\
\text { and serum cortisol } \\
\text { levels during } 1 \mathrm{mg} \text { DST } \\
<3.0 \mu \mathrm{g} / \mathrm{dl}\end{array}$ & Adrenalectomy & $\begin{array}{l}\text { The change in serum cortisol levels during the 1-mg } \\
\text { DST before and after adrenalectomy and histological } \\
\text { characteristics }\end{array}$ & Cohort study \\
\hline Tang et al. (14) & 2018 & China & 414 patients with APA & $\begin{array}{l}\text { Coexistence of APA and subclinical cortisol hypersecretion ( } \mathrm{n}=22 \text {, } \\
\text { serum cortisol levels during } 1 \mathrm{mg} \text { DST }>1.8 \mu \mathrm{g} / \mathrm{dl})\end{array}$ & Clinical and histopathological characteristics & Cross-sectional study \\
\hline Adolf et al. (30) & 2019 & Germany & 73 patients with $\mathrm{PA}^{\mathrm{C}}$ & $\begin{array}{l}\text { Cortisol cosecretion (24-h total glucocorticoid outputs collected by } \\
\text { quantitative gas chromatography-mass spectrometry) }\end{array}$ & Left ventricular hypertrophy & Cohort study \\
\hline Ohno et al. (31) & 2019 & Japan & $\begin{array}{l}527 \text { patients with } \\
\text { bilateral PA. }{ }^{d}\end{array}$ & $\begin{array}{l}\text { Bilateral PA cases with adrenal tumors }(n=196) \text { and without adrenal } \\
\text { tumors }(n=331)\end{array}$ & $\begin{array}{l}\text { Hormone levels including serum cortisol levels during } 1 \\
\text { mg DST and clinical complications }\end{array}$ & Cross-sectional study \\
\hline Kometani et al. (32) & 2019 & Japan & 16 APAs & $\begin{array}{l}\text { Coexistence of APA and subclinical cortisol hypersecretion ( } \mathrm{n}=6 \text {, } \\
\text { serum cortisol levels during } 1 \mathrm{mg} \text { DST and at midnight }>1.8 \mu \mathrm{g} / \mathrm{dll})\end{array}$ & Genetic and epigenetic characteristics & Cross-sectional study \\
\hline Bhatt et al. (33) & 2019 & UK & 25 patients with PA & $\begin{array}{l}\text { Coexistence of PA and subclinical cortisol hypersecretion ( } \mathrm{n}=4 \text {, serum } \\
\text { cortisol levels during } 1 \mathrm{mg} \mathrm{DST}>1.8 \mu \mathrm{g} / \mathrm{dl} \text { ) }\end{array}$ & $\begin{array}{l}\text { Metabolic risk factors (ALT, total cholesterol, HDL, LDL } \\
\text { and mean arterial blood pressure) }\end{array}$ & Cross-sectional study \\
\hline Gerards et al. (10) & 2019 & Germany & 161 patients with $\mathrm{PA}^{\mathrm{C}}$ & $\begin{array}{l}\text { Coexistence of PA and subclinical cortisol hypersecretion ( } \mathrm{n}=125 \text {, } \\
\text { serum cortisol levels during } 1 \mathrm{mg} \text { DST }>1.8 \mu \mathrm{g} / \mathrm{dl} \text {, or late-night } \\
\text { salivary cortisol }>1.45 \mathrm{ng} / \mathrm{ml} \text {, or } 24 \text {-h urinary free cortisol }>150 \mu \mathrm{g} / \\
24 \mathrm{~h} \text { ) }\end{array}$ & $\begin{array}{l}\text { Glucose homeostasis evaluated by the standard oral } \\
\text { glucose tolerance test }\end{array}$ & Cohort study \\
\hline Akehi et al. (11) & 2019 & Japan & $\begin{array}{l}890 \text { patients with PA } \\
\text { who conducted } 1 \mathrm{mg} \\
\text { DST }{ }^{d}\end{array}$ & $\begin{array}{l}\text { Coexistence of PA and subclinical cortisol hypersecretion ( } \mathrm{n}=209 \text {, } \\
\text { serum cortisol levels during } 1 \mathrm{mg} \mathrm{DST}>1.8 \mu \mathrm{g} / \mathrm{dl} \text { ) }\end{array}$ & Prevalence of diabetes & Cross-sectional study \\
\hline Handgriff et al. (34) & 2020 & Germany & 97 patients with $\mathrm{PA}^{\mathrm{C}}$ & $\begin{array}{l}\text { Coexistence of PA and subclinical cortisol hypersecretion ( } \mathrm{n}=72 \text {, } \\
\text { serum cortisol levels during } 1 \mathrm{mg} \text { DST }>1.8 \mu \mathrm{g} / \mathrm{dll} \text {, or late-night } \\
\text { salivary cortisol }>1.5 \mathrm{ng} / \mathrm{ml} \text {, or } 24-\mathrm{h} \text { urinary free cortisol }>150 \mu \mathrm{g} / \mathrm{l} \\
\text { [before 2015] or }>83 \mu \mathrm{g} / \text { [ [after 2015]) }\end{array}$ & $\begin{array}{l}\text { The kinetics of anti-thyroid peroxidase and thyroglobulin } \\
\text { antibody before and after the therapy initiation }\end{array}$ & Cohort study \\
\hline O’Toole et al. (35) & 2020 & UK & 144 patients with PA & $\begin{array}{l}\text { Coexistence of PA and subclinical cortisol hypersecretion ( } \mathrm{n}=21 \text {, } \\
\text { serum cortisol levels during } 1 \mathrm{mg} \text { DST }>1.8 \mu \mathrm{g} / \mathrm{dl})\end{array}$ & $\begin{array}{l}\text { Parameters and interpretation in adrenal venous } \\
\text { sampling }\end{array}$ & Cross-sectional study \\
\hline Peng et al. (15) & 2020 & Taiwan & 82 patients with APA & $\begin{array}{l}\text { Coexistence of APA and subclinical cortisol hypersecretion ( } \mathrm{n}=22 \text {, } \\
\text { serum cortisol levels during } 1 \mathrm{mg} \text { DST }>1.8 \mu \mathrm{g} / \mathrm{dl})\end{array}$ & Clinical and biochemical outcomes after adrenalectomy & Cohort study \\
\hline \multicolumn{7}{|c|}{ 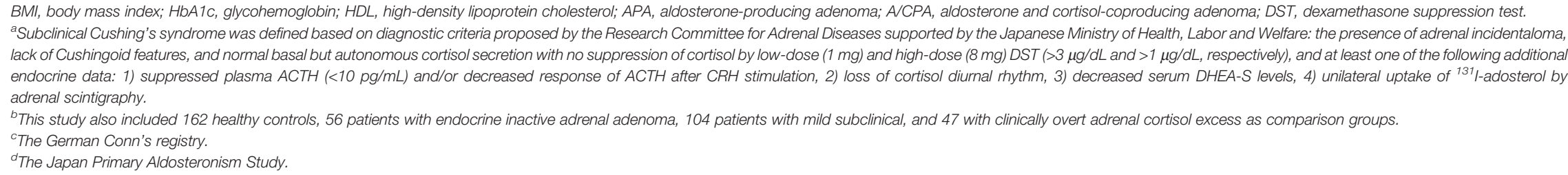 } \\
\hline
\end{tabular}


further understand which AVS parameters are useful for the management of PA with cortisol cosecretion.

Lastly, some studies have investigated the genetic mutation of APA with cortisol cosecretion. A recent study based on a Taiwanese database showed that the presence of KCNJ5 mutations was significantly lower among APA patients with subclinical hypercortisolemia than those without (15). Their immunohistochemistry analysis revealed the lower CYP11B1 expression among KCNJ5 mutated APAs, which generally display a ZF-like phenotype (43). Although the underlying mechanism is unclear, enhanced 18-oxocortisol synthesis in KCNJ5 mutated APAs may contribute to the findings of the lower cortisol levels in the mutated APAs than KCNJ5 wild-type APAs. They also found that patients with subclinical hypercortisolemia and KCNJ5 wild-type APAs exhibited the lowest complete clinical success rate (36.8\%) after adrenalectomy (43), highlighting the importance of detecting these phenotypes for the management of APAs (15). Genetic and epigenetic analysis in a Japanese single-center study showed that APA with hypercortisolemia was not associated with the prevalence of KCNJ5 mutations but associated with lower DNA methylation rate of the CYP11B1 promoter than APA without hypercortisolemia (32). However, this study was based on only 16 APAs and the causal relationship between DNA methylation and the regulation of gene expression is still unclear. Given that some somatic mutations, such as CTNNB1 and PRKACA $(44,45)$, have also been reported in both APAs and CPAs, future research about these mutations and cortisol cosecretion among PA patients would be warranted.

\section{ACTH STIMULATION TEST FOR THE DIAGNOSIS OF PRIMARY ALDOSTERONISM}

Aldosterone production from the adrenal glomerulosa is regulated by ACTH as well as potassium, angiotensin II, and serotonin (46). Previous biological studies showed that acute ACTH administration increases plasma aldosterone through binding to MC2R (47), suggesting that ACTH may play a role in inappropriate hypersecretion of aldosterone in some PAs (16). A previous study showed aldosterone responsiveness to even very low dose ACTH (i.e., $0.003 \mathrm{IU}$ ), followed by a treadmill test (48). Since the first report of the ACTH stimulation test for patients with PA in 1978 (5), several epidemiological studies have investigated whether the ACTH stimulation test is useful to diagnose PA or its subtype. We conducted literature searches between January 2000 and November 2020 using the electronic databases MEDLINE and EMBASE for cohort studies investigating the cortisol cosecretion in primary aldosteronism. The following search terms were applied: ("ACTH" OR "adrenocorticotropic hormone" OR "corticotropin") AND ("primary aldosteronism" OR "aldosterone-producing adenoma"). We extracted the following information: first author name, publication year, region of study, populations, exposures/comparators, outcomes, and study design. We restricted studies to those with a sample size $\geq 10$ (to avoid case reports) and written in English. Flow of studies through review and summary of the identified 9 studies are shown in Figure 1B and Table 2 (17-19, 49-54).

In 1995, Stowasser et al. reported higher plasma aldosterone response after ACTH stimulation under dexamethasone suppression among angiotensin II responsive APA $(n=16)$ or angiotensin II unresponsive APA $(\mathrm{n}=11)$ compared with BAH $(n=19)$ (55). Although several studies mostly from Japan have shown moderate to high predictive performance (area under the curve [AUC] ranges from 0.70 to 0.95 ) to differentiate APA from BAH by using ACTH stimulation test $(17,18,49-52,54)$ over the last two decades, these findings are not comparable across studies due to the lack of uniformity in protocols in each study (e.g., ACTH dosage, with or without $1 \mathrm{mg}$ DST, different cut-off time or values, etc), inconsistent definition of outcomes (e.g., diagnosis of APA and BAH based on AVS, histopathological findings, etc.), and study design (sample size, single-center or multi-center, prospective or retrospective, etc.). These limitations have made the clinical usefulness of the ACTH stimulation test inconclusive. For example, we previously demonstrated that the predictive performance of the ACTH stimulation test for subtype diagnosis of PA could vary by the definition of lateralization during AVS with ACTH stimulation (51); i.e., whether evaluating the ratio of aldosterone to cortisol at adrenal central veins (21) or evaluating the absolute value of aldosterone at adrenal tributary veins (56). Compared with the subtype diagnosis of PA, the evidence about the definite diagnosis of PA has been very limited and has not been consistent (49-51). As the current literature on this topic is mostly based on small sample size and a single-center or a specific country, further investigations with larger sample size from multi-center cohort are needed to understand whether the ACTH stimulation test should be routinely included during the process of PA diagnosis and which parameters are useful to minimize the use of AVS which is more invasive and challenging than peripheral blood examination.

Whether ACTH stimulation is recommended during AVS for subtype diagnosis or not is another important topic in PA management. ACTH stimulation may maximize cortisol secretion from the adrenal gland and minimize the pulsatile adrenocortical hormone secretion. A recent meta-analysis showed that AVS with ACTH stimulation did not significantly reduce the number of incorrect lateralization but significantly reduced the number of unsuccessful cannulations compared with AVS without ACTH stimulation (57). However, the evidence is still limited and under debate which is beyond the scope of this review and can be found in prior review literature (58).

Aldosterone responsiveness to ACTH may vary by a genetic mutation. Our group recently reported a larger decrease in aldosterone levels during $1 \mathrm{mg}$ DST among KCNJ5 mutated APAs than KCNJ5 wild-type APAs, indicating that the ACTH pathway may be more sensitive and activated among KCNJ5 mutated APAs (20). We also reported that aldosterone levels were more responsive to ACTH stimulation among APAs with somatic mutation of CACNA1D than those without the mutation (59). APAs with these mutations are likely to consist of ZF-like clear cells than others, and ZF cells are responsible for cortisol secretion and express a higher level of ACTH receptor than ZG cells $(59,60)$. Given that nearly $90 \%$ of APAs had somatic 


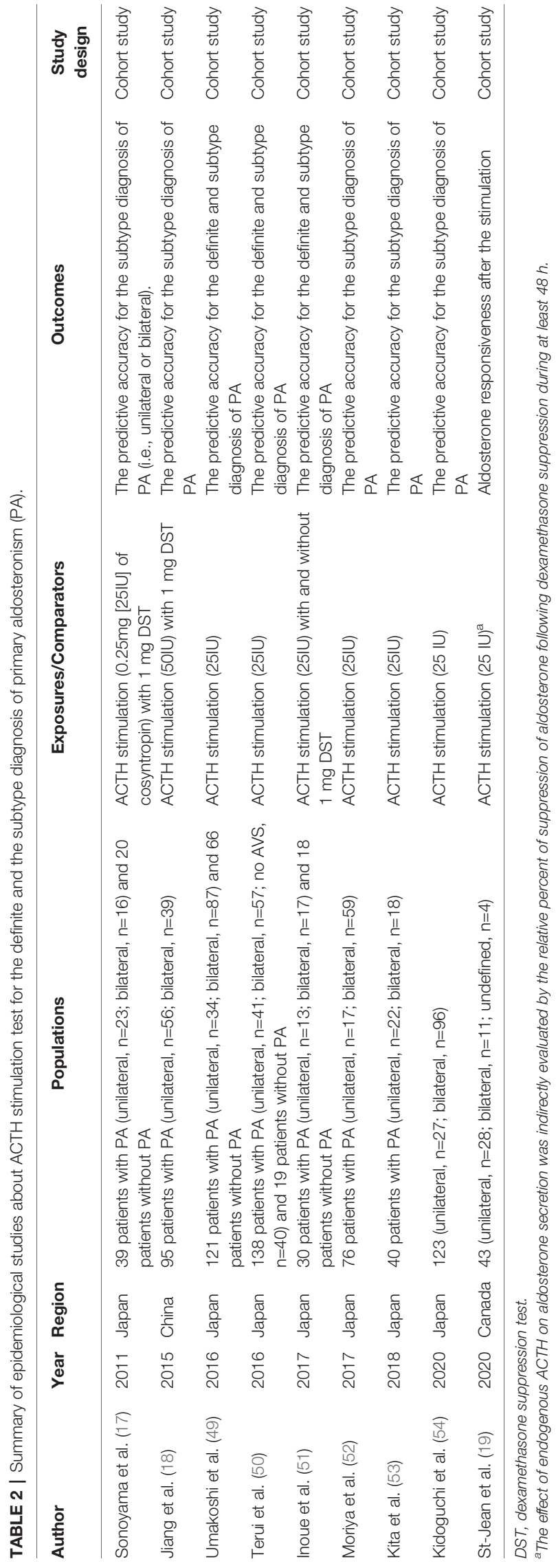

mutations based on the CYP11B2 immunohistochemistryguided, full gene-based next-generation sequencing (61-63), future multi-center studies are warranted to identify the heterogeneous aldosterone response to synthetic ACTH by these genetic mutations.

\section{BIASES IN EPIDEMIOLOGICAL STUDIES RELATED TO PRIMARY ALDOSTERONISM}

Although these previous studies were well-conducted and prismatic, they have suffered from biases that are sometimes substantial. Herein, we describe the common types of biases (confounding, overadjustment, information bias, selection bias, and sampling bias) in epidemiological studies related to PA by using directed acyclic graphs (DAGs), a graphical tool to represent causal relationships between variables by linking them through single-headed arrows (64). In this section, to make a simple explanation, we assume that the exposure of interest is PA with subclinical hypercortisolemia $(\mathrm{PA} / \mathrm{SH})$ and the outcome of interest is CVD.

\section{Confounding}

In observational studies, we generally cannot rule out the possibility of unmeasured confounding. This is also the case for PA studies when some common causes of PA/SH and CVD are missing (Figure 2A). To minimize this bias, researchers should include variables that are causes of the exposure and related to the outcome, or variables that are causes of the outcome and related to the exposure (65). If some important variables are missing, quantitative bias analysis may need to be considered to investigate the potential impact of uncontrolled confounding by the unmeasured confounders (22). This method can also be applied in the metaanalysis of observational studies $(66,67)$. Calculating E-value is an alternative approach to simply assess how much confounding is needed to explain away the observed association (68). Furthermore, if there is information on a genetic mutation that would affect CVD only through PA/SH phenotype and does not modify the effect of $\mathrm{PA} / \mathrm{SH}$ on CVD, mendelian randomization may be considered to obtain a less biased estimate (69).

\section{Overadjustment and Collider Bias}

Another important point to select covariates in the model is overadjustment. Controlling for intermediate variable such as blood pressure (BP) that lies within the causal pathway between $\mathrm{PA} / \mathrm{SH}$ and CVD would introduce a biased estimate (Figure 2B) because we (i) fail to include the effect of $\mathrm{PA} / \mathrm{SH}$ on CVD that is mediated through $\mathrm{BP}$, and (ii) introduce the nonexistent relationship between $\mathrm{PA} / \mathrm{SH}$ and CVD through uncontrolled BP-CVD confounders by controlling for BP, so-called "collider bias" (70, 71). A data-driven approach (including machine learning algorithms) is sometimes useful to efficiently select covariates included in the model, but does not take account of this bias, and therefore, researchers need to carefully select the potential confounders based on prior knowledge and causal diagram (65). 
A
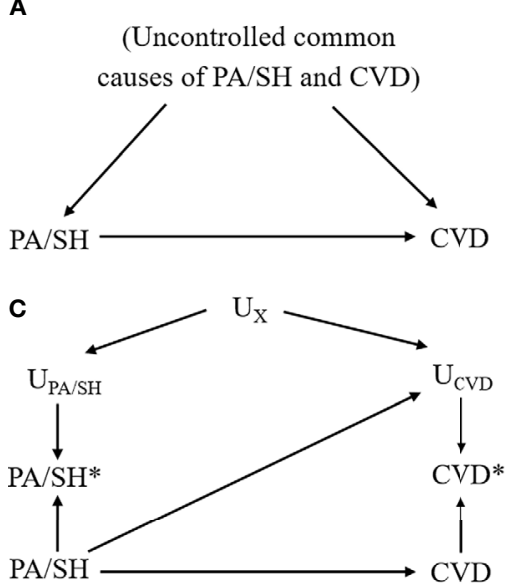

E

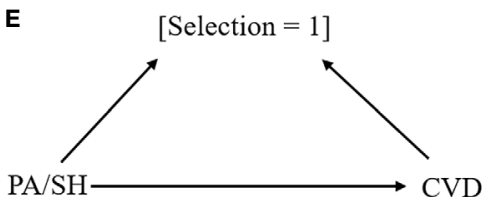

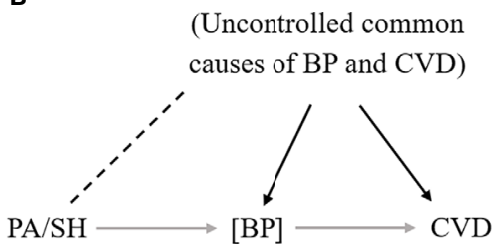

D
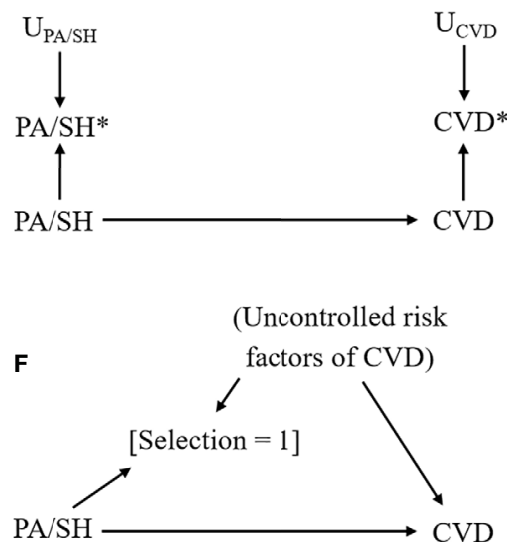

FIGURE 2 | Causal diagram representing each bias scenario in primary aldosteronism study. Notation: Exposure, primary aldosteronism/subclinical hypercortisolemia (PA/SH); Outcome, cardiovascular disease (CVD); solid arrow from $X$ to $Y$, the causal effect of $X$ on $Y$; $[X]$, conditioning on $X$; dash line between $X$ and $Y$, the nonexistent association between $X$ and $Y$ that could be introduced by conditioning on a variable affected by $X$ and $Y$; $U_{X}$, factors that affect the measurement of $X ; X^{*}$, measured $X$ (which could be different from the actual $X$ ). (A) Confounding bias due to uncontrolled common causes of exposure (PA/SH) and outcome (CVD). (B) Overadjustment and collider bias due to conditioning on an intermediate variable (blood pressure [BP]) that underestimate the total effect of PA SH on CVD and introduce additional bias by linking PAVSH and uncontrolled common causes of BP and CVD (dashed line). (C) Measurement error, dependent (i.e., common factors affect measurements of exposure and outcome) and differential (i.e., exposure affects measurements of outcome, or vice versa). (D) Measurement error, independent and nondifferential. (E) Selection bias due to selecting participants by exposure and outcome status. (F) Selection bias due to loss to follow-up.

When there is an interest to estimate the mediation effect of such intermediate variables for the $\mathrm{PA} / \mathrm{SH}-\mathrm{CVD}$ association, causal mediation analysis may be a powerful tool to answer such research questions. For example, using causal mediation analysis, a recent study showed that elevated BP mediated $45 \%$ of the total association between serum aldosterone levels and coronary artery calcium score among general adults from a multi-ethnic group (72). This finding provided novel insight into both the direct effect (not through BP) and indirect effect (through BP) of elevated aldosterone on subclinical atherosclerosis. Meanwhile, it is also noteworthy that causal mediation analysis requires several strong assumptions and limitations. One of the hurdles of applying this method in PA studies is that relatively large sample size is generally needed to find the significant direct and indirect effect of the exposure on the outcome. Further details on this method can be found in the tutorial and methodological review paper (73).

Related to the overadjustment issue, temporal ordering between exposure, confounders, and outcome is crucial to obtain a less biased estimate. In PA studies, given the negative feedback system of RAAS and the HPA axis, it is often challenging to assume which variables (e.g., biomarker concentrations and medical conditions) occurred first at cross-sectional data. When the variables can be both confounders and mediators, researchers need to carefully interpret the results of stratified analysis by such variables because the seemingly heterogeneous findings could be biased (74). Temporal ordering can be established in longitudinal cohorts where the extraction of biosample and other information precedes the outcome assessment.

\section{Measurement Error (Information Bias)}

Because analyses are generally based on data, we need to be careful about the measurement error or misclassification of variables (23). When exposure status (i.e., whether subjects have $\mathrm{PA} / \mathrm{SH}$ or not) affects the reporting of CVD and/or there are common features between exposure assessment and outcome assessment (e.g., physician diagnosis, subjects' awareness of diseases, subjects' cognitive performance, etc), our estimate would be biased (Figure 2C). For example, this type of bias could easily happen when the exposure assessment involves the results of the ACTH stimulation test in peripheral blood and the outcome assessment involves the results of ACTH stimulation during AVS. Recall bias is a similar type of bias that occurs when $\mathrm{PA} / \mathrm{SH}$ assessment is affected by the presence of CVD. If the error for measured PA/SH status (or CVD status) is independent of the true CVD status (or $\mathrm{PA} / \mathrm{SH}$ status) and there are no 
common features that affect $\mathrm{PA} / \mathrm{SH}$ and $\mathrm{CVD}$ measurement (Figure 2D), the bias is generally considered to be towards the null (i.e., underestimate the effect) particularly when the exposure is binary.

\section{Selection Bias and Loss to Follow-Up}

If the study restricts patients to those with confirmatory tests for $\mathrm{PA} / \mathrm{SH}$ and the selection is also affected by health outcome status or its risk factors (which is often the case in a case-control study), such selection introduces collider bias limiting internal validity (Figure 2E). Another example is a loss to follow-up in a cohort study or a randomized controlled trial when enrollees drop the study due to health conditions related to CVD, as known as attrition bias (Figure 2F). Multiple imputations for missing data or inverse probability weighting approach would be helpful to minimize this type of bias when missing mechanisms can be explained by measured covariates (75).

\section{Sampling Bias}

Sampling bias is also caused by selecting participants that limit the generalizability of the study findings. Sampling bias needs to be considered in almost all studies (including clinical trials) because the study sample is often different from the population to which clinical interventions or guidelines are targeted in the real-world. This bias requires attention in PA studies given the different prevalence of $\mathrm{PA} / \mathrm{SH}$ and somatic mutations in APA across countries $(62,63,76)$; i.e., study results from a specific region may not simply be applied to other regions. Moreover, as shown in our literature review, some topic has tended to be heavily interested in a specific region or country, that also limits the external validity. Recently, the concept and method of generalizability and transportability have received renewed interest in causal inference literature to extend the study findings to the target population $(77,78)$.

\section{CONCLUSION}

In this review paper, we summarized the current state of knowledge about primary aldosteronism with cortisol cosecretion and the role of ACTH stimulation test in PA diagnosis. In summary, there is increasing evidence about the relatively high prevalence of cortisol cosecretion in PA and its potential influence on adverse health outcomes. Further studies are warranted to clarify whether there is a clinically useful cut-off points for cortisol cosecretion (i.e., serum cortisol levels during

\section{REFERENCES}

1. Hundemer GL, Curhan GC, Yozamp N, Wang M, Vaidya A. Cardiometabolic outcomes and mortality in medically treated primary aldosteronism: a retrospective cohort study. Lancet Diabetes Endocrinol (2018) 6:51-9. doi: 10.1016/S2213-8587(17)30367-4

2. Mulatero P, Monticone S, Bertello C, Viola A, Tizzani D, Iannaccone A, et al. Long-term cardio- and cerebrovascular events in patients with primary aldosteronism. J Clin Endocrinol Metab (2013) 98:4826-33. doi: 10.1210/ jc.2013-2805 the 1-mg DST) for the PA management. The clinical usefulness of the ACTH stimulation test for PA (subtype) diagnosis has been suggested by some studies, but the evidence is limited to make a specific recommendation in clinical practice. Multicenter studies using the uniform protocol and consistent definition are needed to produce robust evidence on this topic. Moreover, heterogeneous response to ACTH signaling among patients with PA according to genetic mutations should be further investigated. We also explained the common type of biases in these epidemiological studies and also introduced some advanced methods to minimize the biases. As these epidemiological perspectives have not sufficiently been considered in PA-related literature, we hope this review would contribute to a better understanding of possible biases in epidemiological studies and would help clinicians and researchers to produce further robust epidemiological evidence and balanced discussion on these topics.

\section{DATA AVAILABILITY STATEMENT}

The original contributions presented in the study are included in the article/supplementary material. Further inquiries can be directed to the corresponding author.

\section{AUTHOR CONTRIBUTIONS}

Conceptualization, KI, TK, and TN. Methodology, KI. Formal analysis, KI. Investigation, KI, TK, and TN. Writing-original draft preparation, KI. Writing - review and editing, KI, TK, YT, JS, MO, and TN. Visualization, KI. Supervision, TN. All authors contributed to the article and approved the submitted version.

\section{FUNDING}

KI was supported by the National Institutes of Health (NIH)/ NIDDK grant F99DK126119, Toffler award at UCLA, and the Honjo International Foundation Scholarship. This article does not necessarily represent the views and policies of the NIH. The funders had no role in the design and conduct of the study, collection, management, analysis, and interpretation of the data, preparation, review, or approval of the manuscript, and decision to submit the manuscript for publication.

3. Catena C, Colussi G, Nadalini E, Chiuch A, Baroselli S, Lapenna R, et al. Cardiovascular outcomes in patients with primary aldosteronism after treatment. Arch Intern Med (2008) 168:80-5. doi: 10.1001/archinternmed.2007.33

4. Rossi GP, Bernini G, Desideri G, Fabris B, Ferri C, Giacchetti G, et al. Renal damage in primary aldosteronism: results of the PAPY Study. Hypertension (2006) 48:232-8. doi: 10.1161/01.HYP.0000230444.01215.6a

5. Kem DC, Weinberger MH, Higgins JR, Kramer NJ, Gomez-Sanchez C, Holland OB. Plasma aldosterone response to ACTH in primary aldosteronism and in patients with low renin hypertension. J Clin Endocrinol Metab (1978) 46:552-60. doi: 10.1210/jcem-46-4-552 
6. Arlt W, Lang K, Sitch AJ, Dietz AS, Rhayem Y, Bancos I, et al. Steroid metabolome analysis reveals prevalent glucocorticoid excess in primary aldosteronism. JCI Insight (2017) 2:e93136. doi: 10.1172/jci.insight.93136

7. Hanslik G, Wallaschofski H, Dietz A, Riester A, Reincke M, Allolio B, et al. Increased prevalence of diabetes mellitus and the metabolic syndrome in patients with primary aldosteronism of the German Conn's Registry. Eur J Endocrinol (2015) 173:665-75. doi: 10.1530/EJE-15-0450

8. Beuschlein F, Reincke M, Arlt W. The impact of Connshing's syndrome mild cortisol excess in primary aldosteronism drives diabetes risk. J Hypertens (2017) 35:2548. doi: 10.1097/HJH.0000000000001550

9. Wu V-C, Chueh S-CJ, Chen L, Chang C-H, Hu Y-H, Lin Y-H, et al. Risk of newonset diabetes mellitus in primary aldosteronism: a population study over 5 years. J Hypertens (2017) 35:1698-708. doi: 10.1097/HJH.0000000000001361

10. Gerards J, Heinrich DA, Adolf C, Meisinger C, Rathmann W, Sturm L, et al. Impaired Glucose Metabolism in Primary Aldosteronism Is Associated With Cortisol Cosecretion. J Clin Endocrinol Metab (2019) 104:3192-202. doi: $10.1210 /$ jc.2019-00299

11. Akehi Y, Yanase T, Motonaga R, Umakoshi H, Tsuiki M, Takeda Y, et al. High Prevalence of Diabetes in Patients With Primary Aldosteronism (PA) Associated With Subclinical Hypercortisolism and Prediabetes More Prevalent in Bilateral Than Unilateral PA: A Large, Multicenter Cohort Study in Japan. Diabetes Care (2019) 42:938-45. doi: 10.2337/dc18-1293

12. Hiraishi K, Yoshimoto T, Tsuchiya K, Minami I, Doi M, Izumiyama H, et al. Clinicopathological features of primary aldosteronism associated with subclinical Cushing's syndrome. Endocr J (2011) 58:543-51. doi: 10.1507/ endocr.j.k10e-402

13. Fujimoto K, Honjo S, Tatsuoka H, Hamamoto Y, Kawasaki Y, Matsuoka A, et al. Primary aldosteronism associated with subclinical Cushing syndrome. $J$ Endocrinol Invest (2013) 36:564-7. doi: 10.3275/8818

14. Tang L, Li X, Wang B, Ma X, Li H, Gao Y, et al. Clinical Characteristics of Aldosterone- and Cortisol-Coproducing Adrenal Adenoma in Primary Aldosteronism. Int J Endocrinol (2018) 2018:4920841. doi: 10.1155/2018/ 4920841

15. Peng K-Y, Liao H-W, Chan C-K, Lin W-C, Yang S-Y, Tsai Y-C, et al. Presence of Subclinical Hypercortisolism in Clinical Aldosterone-Producing Adenomas Predicts Lower Clinical Success. Hypertension (2020) 76:1537-44. doi: 10.1161/HYPERTENSIONAHA.120.15328

16. Funder JW. The Potential of ACTH in the Genesis of Primary Aldosteronism. Front Endocrinol (Lausanne) (2016) 7:40. doi: 10.3389/fendo.2016.00040

17. Sonoyama T, Sone M, Miyashita K, Tamura N, Yamahara K, Park K, et al. Significance of adrenocorticotropin stimulation test in the diagnosis of an aldosterone-producing adenoma. J Clin Endocrinol Metab (2011) 96:2771-8. doi: 10.1210/jc.2011-0573

18. Jiang Y, Zhang C, Wang W, Su T, Zhou W, Jiang L, et al. Diagnostic value of ACTH stimulation test in determining the subtypes of primary aldosteronism. J Clin Endocrinol Metab (2015) 100:1837-44. doi: 10.1210/jc.2014-3551

19. St-Jean M, Bourdeau I, Martin M, Lacroix A. Aldosterone is Aberrantly Regulated by Various Stimuli in a High Proportion of Patients with Primary Aldosteronism. J Clin Endocrinol Metab (2021) 106:e45-60. doi: 10.1210/ clinem/dgaa703

20. Inoue K, Yamazaki Y, Kitamoto T, Hirose R, Saito J, Omura M, et al. Aldosterone Suppression by Dexamethasone in Patients with KCNJ5Mutated Aldosterone-producing Adenoma. J Clin Endocrinol Metab (2018) 103:3477-85. doi: 10.1210/jc.2018-00738

21. Funder JW, Carey RM, Mantero F, Murad MH, Reincke M, Shibata H, et al. The Management of Primary Aldosteronism: Case Detection, Diagnosis, and Treatment: An Endocrine Society Clinical Practice Guideline. J Clin Endocrinol Metab (2016) 101:1889-916. doi: 10.1210/jc.2015-4061

22. Arah OA. Bias Analysis for Uncontrolled Confounding in the Health Sciences. Annu Rev Public Health (2017) 38:23-38. doi: 10.1146/annurev-publhealth032315-021644

23. Hernán MA, Cole SR. Invited Commentary: Causal diagrams and measurement bias. Am J Epidemiol (2009) 170:959-962; discussion 963964. doi: 10.1093/aje/kwp293

24. Hernán MA, Hernández-Díaz S, Robins JM. A structural approach to selection bias. Epidemiology (2004) 15:615-25. doi: 10.1097/01.ede.0000135174.63482.43

25. Fischer E, Adolf C, Pallauf A, Then C, Bidlingmaier M, Beuschlein F, et al. Aldosterone excess impairs first phase insulin secretion in primary aldosteronism. J Clin Endocrinol Metab (2013) 98:2513-20. doi: 10.1210/ jc.2012-3934

26. Colussi G, Catena C, Lapenna R, Nadalini E, Chiuch A, Sechi LA. Insulin resistance and hyperinsulinemia are related to plasma aldosterone levels in hypertensive patients. Diabetes Care (2007) 30:2349-54. doi: 10.2337/dc07-0525

27. Nakajima Y, Yamada M, Taguchi R, Satoh T, Hashimoto K, Ozawa A, et al. Cardiovascular complications of patients with aldosteronism associated with autonomous cortisol secretion. J Clin Endocrinol Metab (2011) 96:2512-8. doi: 10.1210/jc.2010-2743

28. Fallo F, Bertello C, Tizzani D, Fassina A, Boulkroun S, Sonino N, et al. Concurrent primary aldosteronism and subclinical cortisol hypersecretion: a prospective study. J Hypertens (2011) 29:1773-7. doi: 10.1097/HJH.0b013e3 $2834937 f 3$

29. Inoue K, Yamazaki Y, Tsurutani Y, Suematsu S, Sugisawa C, Saito J, et al. Evaluation of Cortisol Production in Aldosterone-Producing Adenoma. Horm Metab Res (2017) 49:847-53. doi: 10.1055/s-0043-119878

30. Adolf C, Köhler A, Franke A, Lang K, Riester A, Löw A, et al. Cortisol Excess in Patients With Primary Aldosteronism Impacts Left Ventricular Hypertrophy. J Clin Endocrinol Metab (2018) 103:4543-52. doi: 10.1210/ jc.2018-00617

31. Ohno Y, Sone M, Inagaki N, Takeda Y, Kurihara I, Tsuiki M, et al. Latent Autonomous Cortisol Secretion From Apparently Nonfunctioning Adrenal Tumor in Nonlateralized Hyperaldosteronism. J Clin Endocrinol Metab (2019) 104:4382-9. doi: 10.1210/jc.2018-02790

32. Kometani M, Yoneda T, Demura M, Aono D, Gondoh Y, Karashima S, et al. Genetic and epigenetic analyses of aldosterone-producing adenoma with hypercortisolemia. Steroids (2019) 151:108470. doi: 10.1016/j.steroids.2019.108470

33. Bhatt PS, Sam AH, Meeran KM, Salem V. The relevance of cortisol cosecretion from aldosterone-producing adenomas. Hormones (Athens) (2019) 18:307-13. doi: 10.1007/s42000-019-00114-8

34. Handgriff L, Adolf C, Heinrich DA, Braun L, Nirschl N, Sturm L, et al. The Impact of Glucocorticoid Co-Secretion in Primary Aldosteronism on Thyroid Autoantibody Titers During the Course of Disease. Horm Metab Res (2020) 52:404-11. doi: 10.1055/a-1164-1944

35. O’Toole SM, Sze W-CC, Chung T-T, Akker SA, Druce MR, Waterhouse M, et al. Low-grade Cortisol Cosecretion Has Limited Impact on ACTHstimulated AVS Parameters in Primary Aldosteronism. J Clin Endocrinol Metab (2020) 105:e3776-84. doi: 10.1210/clinem/dgaa519

36. Piaditis GP, Kaltsas GA, Androulakis II, Gouli A, Makras P, Papadogias D, et al. High prevalence of autonomous cortisol and aldosterone secretion from adrenal adenomas. Clin Endocrinol (Oxf) (2009) 71:772-8. doi: 10.1111/ j.1365-2265.2009.03551.x

37. Gomez-Sanchez CE, Qi X, Velarde-Miranda C, Plonczynski MW, Parker CR, Rainey W, et al. Development of monoclonal antibodies against human CYP11B1 and CYP11B2. Mol Cell Endocrinol (2014) 383:111-7. doi: 10.1016/ j.mce.2013.11.022

38. Nakamura Y, Maekawa T, Felizola SJA, Satoh F, Qi X, Velarde-Miranda C, et al. Adrenal CYP11B1/2 expression in primary aldosteronism: immunohistochemical analysis using novel monoclonal antibodies. Mol Cell Endocrinol (2014) 392:73-9. doi: 10.1016/j.mce.2014.05.002

39. Fallo F, Castellano I, Gomez-Sanchez CE, Rhayem Y, Pilon C, Vicennati V, et al. Histopathological and genetic characterization of aldosterone-producing adenomas with concurrent subclinical cortisol hypersecretion: a case series. Endocrine (2017) 58:503-12. doi: 10.1007/s12020-017-1295-4

40. Gioco F, Seccia TM, Gomez-Sanchez EP, Rossi GP, Gomez-Sanchez CE. Adrenal histopathology in primary aldosteronism: is it time for a change? Hypertension (2015) 66:724-30. doi: 10.1161/HYPERTENSIONAHA.115.05873

41. Mihailidou AS, Loan Le TY, Mardini M, Funder JW. Glucocorticoids activate cardiac mineralocorticoid receptors during experimental myocardial infarction. Hypertension (2009) 54:1306-12. doi: 10.1161/HYPERTENSIONAHA. 109.136242

42. Goupil R, Wolley M, Ahmed AH, Gordon RD, Stowasser M. Does concomitant autonomous adrenal cortisol overproduction have the potential to confound the interpretation of adrenal venous sampling in primary aldosteronism? Clin Endocrinol (Oxf) (2015) 83:456-61. doi: 10.1111/cen.12750

43. Mulatero P, Monticone S, Rainey WE, Veglio F, Williams TA. Role of KCNJ5 in familial and sporadic primary aldosteronism. Nat Rev Endocrinol (2013) 9:104-12. doi: 10.1038/nrendo.2012.230 
44. Wu V-C, Wang S-M, Chueh S-CJ, Yang S-Y, Huang K-H, Lin Y-H, et al. The prevalence of CTNNB1 mutations in primary aldosteronism and consequences for clinical outcomes. Sci Rep (2017) 7:39121. doi: 10.1038/srep39121

45. Rhayem Y, Perez-Rivas LG, Dietz A, Bathon K, Gebhard C, Riester A, et al. PRKACA Somatic Mutations Are Rare Findings in Aldosterone-Producing Adenomas. J Clin Endocrinol Metab (2016) 101:3010-7. doi: 10.1210/jc.2016-1700

46. Young MJ, Funder JW. The renin-angiotensin-aldosterone system in experimental mineralocorticoid-salt-induced cardiac fibrosis. Am J PhysiologyEndocrinology Metab (1996) 271:E883-8. doi: 10.1152/ajpendo.1996.271.5.E883

47. Young M, Funder JW. Eplerenone, But Not Steroid Withdrawal, Reverses Cardiac Fibrosis in Deoxycorticosterone/ Salt-Treated Rats. Endocrinology (2004) 145:3153-7. doi: 10.1210/en.2004-0005

48. Markou A, Sertedaki A, Kaltsas G, Androulakis II, Marakaki C, Pappa T, et al. Stress-induced Aldosterone Hyper-Secretion in a Substantial Subset of Patients With Essential Hypertension. J Clin Endocrinol Metab (2015) 100:2857-64. doi: 10.1210/jc.2015-1268

49. Umakoshi H, Xiaomei Y, Ichijo T, Kamemura K, Matsuda Y, Fujii Y, et al. Reassessment of the cosyntropin stimulation test in the confirmatory diagnosis and subtype classification of primary aldosteronism. Clin Endocrinol (Oxf) (2017) 86:170-6. doi: 10.1111/cen.13167

50. Terui K, Kageyama K, Nigawara T, Moriyama T, Sakihara S, Takayasu S, et al. Evaluation of the (1-24) adrenocorticotropin stimulation test for the diagnosis of primary aldosteronism. J Renin Angiotensin Aldosterone Syst (2016) 17:1470320315625703. doi: 10.1177/1470320315625703

51. Inoue K, Omura M, Sugisawa C, Tsurutani Y, Saito J, Nishikawa T. Clinical Utility of the Adrenocorticotropin Stimulation Test with/without Dexamethasone Suppression for Definitive and Subtype Diagnosis of Primary Aldosteronism. Int J Mol Sci (2017) 18:948. doi: 10.3390/ijms18050948

52. Moriya A, Yamamoto M, Kobayashi S, Nagamine T, Takeichi-Hattori N, Nagao M, et al. ACTH stimulation test and computed tomography are useful for differentiating the subtype of primary aldosteronism. Endocrine J (2017) 64:65-73. doi: 10.1507/endocrj.EJ16-0297

53. Kita T, Furukoji E, Sakae T, Kitamura K. Efficient screening of patients with aldosterone-producing adenoma using the ACTH stimulation test. Hypertension Res (2019) 42:801-6. doi: 10.1038/s41440-018-0191-5

54. Kidoguchi S, Sugano N, Kawauchi R, Nakashima D, Hayashi-Ishikawa N, Tokudome G, et al. Evaluation of various confirmatory tests for the diagnosis of aldosterone-producing adenoma. J Renin Angiotensin Aldosterone Syst (2020) 21:1470320320919610. doi: 10.1177/1470320320919610

55. Stowasser M, Klemm SA, Tunny TJ, Gordon RD. Plasma aldosterone response to ACTH in subtypes of primary aldosteronism. Clin Exp Pharmacol Physiol (1995) 22:460-2. doi: 10.1111/j.1440-1681.1995.tb02044.x

56. Kitamoto T, Kitamoto KK, Omura M, Takiguchi T, Tsurutani Y, Kubo H, et al. Precise Mapping of Intra-Adrenal Aldosterone Activities Provides a Novel Surgical Strategy for Primary Aldosteronism. Hypertension (2020) 76:976-84. doi: 10.1161/HYPERTENSIONAHA.119.14341.

57. Laurent I, Astère M, Zheng F, Chen X, Yang J, Cheng Q, et al. Adrenal venous sampling with or without adrenocorticotropic hormone stimulation: A metaanalysis. J Clin Endocrinol Meta (2018) 104:1060-8. doi: 10.1210/jc.2018-01324

58. Deinum J, Groenewoud H, van der Wilt GJ, Lenzini L, Rossi GP. Adrenal venous sampling: cosyntropin stimulation or not? Eur J Endocrinol (2019) 181:D15-26. doi: 10.1530/EJE-18-0844

59. Kitamoto T, Suematsu S, Yamazaki Y, Nakamura Y, Sasano H, Matsuzawa Y, et al. Clinical and Steroidogenic Characteristics of Aldosterone-Producing Adenomas With ATPase or CACNA1D Gene Mutations. J Clin Endocrinol Metab (2016) 101:494-503. doi: 10.1210/jc.2015-3284

60. Azizan EAB, Lam BYH, Newhouse SJ, Zhou J, Kuc RE, Clarke J, et al. Microarray, qPCR, and KCNJ5 sequencing of aldosterone-producing adenomas reveal differences in genotype and phenotype between zona glomerulosa- and zona fasciculata-like tumors. J Clin Endocrinol Metab (2012) 97:E819-829. doi: 10.1210/jc.2011-2965

61. Nanba K, Omata K, Else T, Beck PCC, Nanba AT, Turcu AF, et al. Targeted Molecular Characterization of Aldosterone-Producing Adenomas in White Americans. J Clin Endocrinol Metab (2018) 103:3869-76. doi: 10.1210/ jc.2018-01004
62. Nanba K, Omata K, Gomez-Sanchez CE, Stratakis CA, Demidowich AP, Suzuki M, et al. Genetic Characteristics of Aldosterone-Producing Adenomas in Blacks. Hypertension (2019) 73:885-92. doi: 10.1161/HYPERTENSIONAHA. 118.12070

63. Nanba K, Yamazaki Y, Bick N, Onodera K, Tezuka Y, Omata K, et al. Prevalence of Somatic Mutations in Aldosterone-Producing Adenomas in Japanese Patients. J Clin Endocrinol Metab (2020) 105:e4066-73. doi: 10.1210/ clinem/dgaa595

64. Greenland S, Pearl J, Robins JM. Causal diagrams for epidemiologic research. Epidemiology (1999) 10(1):37-48.

65. VanderWeele TJ. Principles of confounder selection. Eur J Epidemiol (2019) 34:211-9. doi: 10.1007/s10654-019-00494-6

66. Goto A, Arah OA, Goto M, Terauchi Y, Noda M. Severe hypoglycaemia and cardiovascular disease: systematic review and meta-analysis with bias analysis. BMJ (2013) 347:f4533. doi: 10.1136/bmj.f4533

67. Inoue K, Beekley J, Goto A, Jeon CY, Ritz BR. Depression and cardiovascular disease events among patients with type 2 diabetes: A systematic review and meta-analysis with bias analysis. J Diabetes Complicat (2020) 34:107710. doi: 10.1016/j.jdiacomp.2020.107710

68. VanderWeele TJ, Ding P. Sensitivity Analysis in Observational Research: Introducing the E-Value. Ann Intern Med (2017) 167:268-74. doi: 10.7326/ M16-2607

69. Davies NM, Holmes MV, Davey Smith G. Reading Mendelian randomisation studies: a guide, glossary, and checklist for clinicians. BMJ (2018) 362:k601. doi: 10.1136/bmj.k601

70. Greenland S. Quantifying biases in causal models: classical confounding vs collider-stratification bias. Epidemiology (2003) 14:300-6. doi: 10.1097/ 01.EDE.0000042804.12056.6C

71. Cole SR, Platt RW, Schisterman EF, Chu H, Westreich D, Richardson D, et al. Illustrating bias due to conditioning on a collider. Int J Epidemiol (2010) 39:417-20. doi: 10.1093/ije/dyp334

72. Inoue K, Goldwater D, Allison M, Seeman T, Kestenbaum BR, Watson KE. Serum Aldosterone Concentration, Blood Pressure, and Coronary Artery Calcium: The Multi-Ethnic Study of Atherosclerosis. Hypertension (2020) 76:113-20. doi: 10.1161/HYPERTENSIONAHA.120.15006

73. VanderWeele TJ. Mediation Analysis: A Practitioner's Guide. Annu Rev Public Health (2016) 37:17-32. doi: 10.1146/annurev-publhealth-032315021402

74. Inoue K, Goto A, Sugiyama T, Ramlau-Hansen CH, Liew Z. The ConfounderMediator Dilemma: Should We Control for Obesity to Estimate the Effect of Perfluoroalkyl Substances on Health Outcomes? Toxics (2020) 8:125. doi: $10.3390 /$ toxics 8040125

75. Howe LD, Tilling K, Galobardes B, Lawlor DA. Loss to Follow-up in Cohort Studies. Epidemiology (2013) 24:1-9. doi: 10.1097/EDE.0b013e31827623b1

76. Fernandes-Rosa FL, Williams TA, Riester A, Steichen O, Beuschlein F, Boulkroun S, et al. Genetic spectrum and clinical correlates of somatic mutations in aldosterone-producing adenoma. Hypertension (2014) 64:35461. doi: 10.1161/HYPERTENSIONAHA.114.03419

77. Bareinboim E, Pearl J. Causal inference and the data-fusion problem. PNAS (2016) 113:7345-52. doi: 10.1073/pnas.1510507113

78. Dahabreh IJ, Robertson SE, Steingrimsson JA, Stuart EA, Hernán MA. Extending inferences from a randomized trial to a new target population. Stat Med (2020) 39:1999-2014. doi: 10.1002/sim.8426

Conflict of Interest: The authors declare that the research was conducted in the absence of any commercial or financial relationships that could be construed as a potential conflict of interest.

Copyright (c) 2021 Inoue, Kitamoto, Tsurutani, Saito, Omura and Nishikawa. This is an open-access article distributed under the terms of the Creative Commons Attribution License (CC BY). The use, distribution or reproduction in other forums is permitted, provided the original author(s) and the copyright owner(s) are credited and that the original publication in this journal is cited, in accordance with accepted academic practice. No use, distribution or reproduction is permitted which does not comply with these terms. 